\title{
Pyrolysis and Devolatilization of High-Density Polyethylene
}

\author{
MORGAN C. BRUNS, and OFODIKE A. EZEKOYE \\ Department of Mechanical Engineering \\ The University of Texas at Austin \\ 1 University Station, C2200 \\ Austin, TX 78712 USA
}

\begin{abstract}
A simple model for combined pyrolysis and devolatilization of high-density polyethylene (HDPE) is developed and studied. The model parameters are estimated from available literature data, and the resulting predictions are compared with a single thermogravimetric experiment. It is found that the bubble loss model makes a better prediction than a critical carbon number loss model. As expected, the bubble loss model is seen to lose validity when the sample becomes predominantly composed of volatile species.
\end{abstract}

KEYWORDS: pyrolysis, modeling, devolatilization, flammability, polyethylene, TGA.

\section{NOMENCLATURE LISTING}

$\begin{array}{llll}A_{s} & \text { sample surface area }\left(\mathrm{mm}^{2}\right) & \text { Greek } & \\ D & \text { diffusivity }\left(\mathrm{cm}^{2} / \mathrm{s}\right) & \alpha & \text { growth coefficient }\left(\mathrm{kg}^{2 / 3} / \mathrm{s}\right) \\ f & \text { free-volume fraction } & \beta & \text { velocity coefficient }\left(\mathrm{m} / \mathrm{kg}^{2 / 3} \cdot \mathrm{s}\right) \\ g & \text { gravitational constant }\left(\mathrm{m}^{2} / \mathrm{s}\right) & \mu & \text { melt viscosity }(\mathrm{kg} / \mathrm{m}-\mathrm{s}) \\ H & \text { sample height }(\mathrm{mm}) & \rho & \text { density }\left(\mathrm{kg} / \mathrm{m}^{3}\right) \\ J & \text { nucleation rate }\left(1 / \mathrm{cm}^{3} \cdot \mathrm{s}\right) & \sigma & \text { surface tension }(\mathrm{mN} / \mathrm{m}) \\ k & \text { scission rate }\left(\mathrm{s}^{-1}\right) & \tau_{b} & \text { bubble lifespan }(\mathrm{s}) \\ K_{\phi} & \text { Henry's law coefficient } & \phi & \text { volume fraction } \\ m & \text { mass }(\mathrm{mg}) & \chi & \text { interaction parameter } \\ M & \text { molecular weight }(\mathrm{kg} / \mathrm{mol}) & \text { subscripts } \\ n & \text { carbon number } & 1 & \text { volatile species } \\ p_{i} & \text { number of polymers of size } i & 2 & \text { polymer species } \\ P & \text { pressure }(\text { Pa }) & c & \text { critical value } \\ T & \text { temperature }(\mathrm{K}) & e & \text { bubble exiting surface } \\ \bar{v}_{1} & \text { molar volume }\left(\mathrm{cm}^{3} / \mathrm{mol}\right) & n & \text { bubble nucleating } \\ V_{e} & \text { molar volume of end groups } & i & \text { initial value } \\ z & \text { spatial height coordinate }(\mathrm{mm}) & b & \text { bubble (or boiling temperature) }\end{array}$

\section{INTRODUCTION}

Our natural and synthetic environment is composed primarily of polymeric materials. A large portion of these materials are organic thermoplastics which contribute significantly to the fire load of the buildings we occupy. To address this problem, the fire research community has sought to make improvements in building design, sprinkler systems, and firefighting tactics and technology. Another approach is to develop fire resistant (FR) materials by the addition of flame retardants and identifying materials that are inherently less flammable. A material's flammability depends on the rates at which volatile gas products are generated inside the burning solid.

The conversion of condensed phase polymers to gas is critical in predicting both ignition and steady burning of fires. This process can be considered as two coupled steps: (1) pyrolysis - the chemical conversion of polymer chains to successively smaller molecules and (2) devolatilization - the mass transport of the volatiles across the surface of the condensed phase. Step (1) has been considered in the pyrolysis literature, but typically without any consideration of the devolatilization of the pyrolysis products. In this paper, a step is taken towards synthesizing these two steps for the case of the structurally simple thermoplastic, high-density polyethylene (HDPE). Typically, the kinetic rates for polymer degradation are inferred from global thermogravimetric (TGA) experiments. In such cases, the critical assumption is that 
the devolatilization process is significantly faster than the kinetic processes (pyrolysis) so that the effective kinetics derived from the TGA data correspond to the pyrolysis chemistry. The heating rates in typical TGA experiments are significantly slower than the heating rates in fires and other high heat flux thermoplastic degradation processes. Thus, the validity of the TGA results is questionable for higher heating rate conditions. The difficulty in making in situ measurements during thermoplastic degradation has limited the development of first principles models of the degradation processes. Also, the complexity of the problem is a challenge to modeling. Because of the increasing demand for prediction tools to predict ignition and degradation of novel thermoplastics and thermoplastic composites, there is a need for high fidelity degradation models.

The objectives of this paper are (1) to describe a simple model of TGA that couples pyrolysis and devolatilization physics, (2) identify measurements and/or models of the parameters of this model, and (3) to compare the predictions of the model to isothermal TGA experiments of HDPE.

As a starting point towards achieving this goal, a simple isothermal TGA system is considered. The models will be developed in the context of an isothermal polymer melt contained within a TGA sample pan as shown in Fig. 1.

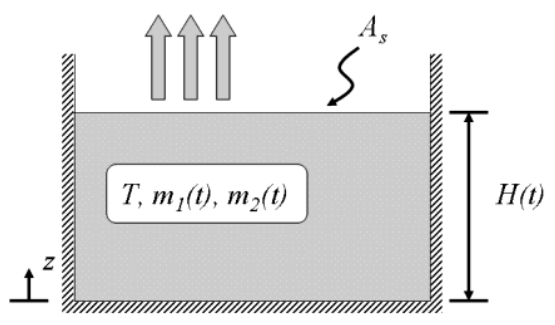

Fig. 1. Diagram of a thermally degrading polymer melt.

The top surface is the only surface through which mass can leave the system. Note that the melt temperature for HDPE is around $138^{\circ} \mathrm{C}$ whereas significant pyrolysis does not occur until around $400{ }^{\circ} \mathrm{C}$ so pyrolysis is always in a liquid state.

A loss model is needed to account for devolatilization. All species devolatilize - though at decreasing rates with increasing carbon number. As time proceeds, pyrolysis increases the amount of volatiles in the melt. Because the sample temperature is assumed to be spatially uniform, the volatile generation will also be spatially uniform. Volatiles at the surface of the sample will devolatilize inducing a concentration gradient in the melt. Diffusion of volatiles to the surface is one possible mechanism for mass loss. However, it is known from observation that bubbling is vigorous at pyrolysis temperatures in HDPE. Bubbling reduces the volatile concentration gradient, and introduces another, presumably dominant, loss mechanism referred to here as bubbling mass loss.

Several authors have considered bubbling mass loss. Wichman [1] used a bubble number conservation equation to study degradation due to an imposed surface heat flux. For the purposes of comparing their kinetic pyrolysis models to experimental GC-MS data, Faravelli et al. [2] developed a simple bubbling loss model. Butler [3] developed models for bubbling in combusting thermoplastic materials and incorporated these models into numerical simulations.

\section{BUBBLING MASS LOSS AND MATERIAL PROPERTY MODELS}

In this section, a general framework is presented for modeling bubbling mass loss. Additionally, an approach for material property characterization is discussed.

Consider a single bubble which nucleates, migrates, grows, and eventually crosses the sample surface. A bubble crossing the surface results in a total mass loss equal to the mass of the gas inside the bubble. In order to determine the mass loss rate for a system with many bubbles, it is necessary to model the mass and number of bubbles crossing the surface per unit of time. Depending on the location of their nucleation, bubbles crossing the surface will have different masses. The total mass loss rate is found by integration over the sample height 


$$
\frac{d m}{d t}=-A_{s} \int_{0}^{H} m_{e}\left(z_{n}\right) J\left(t-\tau_{b}\left(z_{n}\right)\right) d z_{n}
$$

where $m_{e}\left(z_{n}\right)$ and $\tau_{b}\left(z_{n}\right)$ are the mass and lifespan of a bubble exiting the surface at time $t$ and which were nucleated at position $z_{n}$. The nucleation rate, denoted $J$, is the nucleation rate (number of bubbles produced per unit volume per unit second). Models for these three parameters are presented in the following sections.

It is important to clarify the dependence of the model parameters on the other model variables. All properties are ultimately dependent on the thermodynamic state in the melt. The chemical composition is fully characterized by the chain length distribution. It is assumed that this distribution can be approximated by the number average molecular weights (MW) of a notional volatile species, $M_{1}$, and a notional polymer species, $M_{2}$, along with the volume fraction of volatiles, $\phi_{1}$. Since volatility on the thermodynamic state, it is assumed that the average volatile molecular weight only depends on the pressure and temperature, both of which are assumed constant. The molecular weight of the polymer will be modeled by an analytical function of time as discussed in the next section. Finally, a model is needed for the time evolution of the volatile volume fraction. With models for these three quantities it is possible to express the functional dependence of any material property as a function of volatile volume fraction and time only.

\section{PYROLYSIS MODEL}

Before examining the bubble loss models, the process of volatile generation through pyrolysis will be discussed. The chemical mechanism of pyrolysis depends on the material and its thermodynamic state. There is much literature discussing the probable mechanisms of thermoplastic pyrolysis [4-6]. Radical depolymerization is a common description for many thermoplastics. However, it is generally believed that polyethylene pyrolizes according to a random scission mechanism. Random scission is an approximation of radical depolymerization in systems where the unzipping reaction is relatively slow compared to the termination reactions. Since pyrolysis modeling is not the primary objective of this paper, and since polyethylene is the material studied, pyrolysis will be modeled as being a purely random scission mechanism.

Assuming the system is a linear thermoplastic - such as HDPE, the molecules of the system are characterized entirely by their size measured as their carbon number, $n$. HDPE molecules have relatively few side-chain branches and can be approximated as extremely long $n$-alkanes or $n$-alkenes. Since the chain-end hydrogen atoms have a negligible impact on mass for long $n$-alkanes, they will be ignored in the following. Therefore, the MW of a single HDPE molecule is related to its carbon number by $M(n)=M_{0} n$ where $M_{0}=14.0265$ is the molecular weight of $\mathrm{C}_{2}$ unit.

In a sample of HDPE, there is a distribution of species over their lengths. Each molecule of a given length can be considered a separate species. A species is denoted by $\mathrm{P}_{i}$ where the subscript is the carbon number. The random scission of the polymer chains is described by the chemical equation

$$
\mathrm{P}_{i} \stackrel{k(T)}{\longrightarrow} \mathrm{P}_{j}+\mathrm{P}_{i-j}
$$

where $k(T)$ is the temperature dependent reaction rate-specifically, the rate at which all carbon-carbon bonds break. The species concentrations in the sample are governed by the system of ODEs

$$
\frac{d p_{i}}{d t}=k\left[-(i-1) p_{i}+2 \sum_{j=i+1}^{N} p_{j}\right], \quad i=n_{c}, \ldots N
$$

Various forms of this model have been presented and evaluated in, for example, Refs. [4-7]. There is an exact solution for constant temperatures.

For simplification, it will be assumed that there are only two distinct notional species. Species 1 is a volatile molecule, and species 2 is a non-volatile polymer. The masses of these species in the melt are $m_{1}$ 
and $m_{2}$. The dividing line between the species included in the notional volatile and the species included in the notional polymer is represented by a critical carbon number, $n_{c}$.

In TGA modeling, the measured quantity is the mass, but the transport mechanisms by which mass changes are controlled by the number averaged MW of the melt. Analytical expressions for the mass and MW of the polymer melt can be derived from the moment solutions of Eq. 3:

$$
\begin{aligned}
& m_{2}(t)=m_{i}\left[1+n_{c}\left(1-e^{-k t}\right)\right] e^{-k n_{c} t} \\
& M_{2}(t)=M_{0} \frac{\bar{n}_{i}+n_{c}\left(\bar{n}_{i}-n_{c}-1\right)\left(1-e^{-k t}\right)}{\bar{n}_{i}-n_{c}-\left(\bar{n}_{i}-n_{c}-1\right) e^{-k t}}
\end{aligned}
$$

where $m_{i}$ and $\bar{n}_{i}$ are the initial sample mass and number-average carbon number.

Equations 4 and 5 represent a model of isothermal pyrolysis in which the molecules immediately loose membership in the polymer population (species 2) if they are smaller than the critical carbon number $n_{c}$. The newly generated volatile molecules (species 1) are still present in the sample, and it takes time for them to exit the sample surface. In the next section, a loss model is developed to account for the lag in mass loss after pyrolysis has generated volatiles.

\section{BUBBLE DYNAMICS}

The smaller molecules generated by pyrolysis are free to diffuse through the polymer melt. When enough volatile molecules diffuse in to a small region a bubble is born. The bubble will rise due to its buoyancy and grow due to diffusion of volatile molecules to its surface. In this section, submodels for the parameters of Eq. 1 are derived. The nucleation rate is independent of the other submodels, but the mass of exiting bubbles depends upon submodels for the bubble growth rate and migration velocity. All of these models are taken adopted from the literature, and more sophisticated models exist. For the purpose of this paper, the simplest, reasonable submodel for each process was chosen.

\section{Nucleation}

Both homogeneous and heterogeneous nucleation can be modeled as Arrhenius processes where the activation energy is equated to the work required to form a stable bubble. This work is dependent on both the surface tension and the change in pressure in the transition from a liquid to a gas. A general model form from classical nucleation theory is

$$
J=A \sigma^{1 / 2} \exp \frac{-B \sigma^{3}}{T\left(K_{\phi} \phi_{1}-P\right)^{2}}
$$

where $\sigma$ is the bubble surface tension, $K_{\phi}$ is the Henry's law coefficient, $B$ depends upon whether the nucleation is homogeneous or heterogeneous, and $A$ depends upon the relative amount of volatiles if nucleation is heterogeneous. There is a significant literature [8-12] on approximating the values of $A$ and $B$ from more fundamental quantities, but as of yet it does not appear that any one theory is fully adequate.

Heterogeneous nucleation is much more probable at low temperatures. Since the number and geometry of the heterogeneous nucleation sites is unknown, the coefficients $A$ and $B$ will be treated as unknown model parameters.

\section{Growth}

Bubbles grow by the transport of volatile molecules from the melt to the bubble surface and the subsequent devolatilization of the surface volatiles to the interior of the bubble. A simple model for bubble growth is due to Epstein and Plesset [13]. This model assumes that (i) the diffusion is steady state, (ii) the bubble is stationary, (iii) the bubble gas is ideal, (iv) the bubble pressure is constant, (v) the process is isothermal, 
(vi) the pressure inside the bubble is equal to the pressure inside the liquid, and (vii) viscous normal stresses are negligible. As a consequence of assumption (ii), the predicted growth rate is expected to be slower than the actual growth rate.

The Epstein-Plesset model for bubble growth, in terms of the bubble's mass, is

$\dot{m}_{b}=7.796 \frac{D_{1} M_{1}\left(\phi_{1}-P / K_{\phi}\right)}{\bar{v}_{1} \rho_{b}^{1 / 3}} m_{b}^{1 / 3}$

where $D$ is the diffusivity of the volatiles in the condensed phase, $\bar{v}_{1}$ is the molar volume of the volatile species, and $\rho_{b}$ is the density of the volatile gas inside the bubble. In deriving Eq. 7, it was assumed that the volume fraction could be related to the molar concentration through $\phi_{1}=\bar{v}_{1} c_{1}$. Also, the fact that the pressure inside the bubble equals the pressure in the melt implies that the volume fraction of volatile at the surface of the bubble is $P / K_{\phi}$.

Note that since the parameters of Eq. 7 are not dependent upon $m_{b}$, the differential equation is separable. Furthermore, if it is assumed that the composition and material properties are constant over the lifespan of the bubble, then the mass stored in a single bubble as a function of time from its nucleation can be computed as

$m^{2 / 3}(t)=m_{n}^{2 / 3}+\alpha t$

where a growth parameter is defined by

$\alpha \equiv 5.197 \frac{M_{1} D_{1}\left(P_{1}-P\right)}{K_{\phi} \bar{v}_{1} \rho_{b}^{1 / 3}}$

with units of $[\mathrm{mass}]^{2 / 3} /[$ time $]$, and $P_{1}=K_{\phi} \phi_{1}$.

\section{Migration}

The final submodel is for migration velocity. This model is necessary so that the lifespan of the bubble can be computed as a function of its nucleation coordinate. It is assumed that the bubble reaches its equilibrium velocity quickly relative to its growth. If the Reynold's number is small, and the fluid is Newtonian, then the bubble velocity is computed from Stokes' Law as

$\dot{z}=0.08552 \frac{g\left(\rho-\rho_{b}\right)}{\rho_{b}^{2 / 3} \mu} m_{b}^{2 / 3}$

where $\mu$ is the Newtonian viscosity of the melt. The solution of this equation provides information about the lifespan of a single bubble. Substituting Eq. 8 into Eq. 10 and assuming constant properties gives

$\tau_{b}\left(z_{n}\right)=\frac{1}{\alpha}\left[-m_{n}^{2 / 3}+\sqrt{m_{n}^{4 / 3}+\frac{2 \alpha\left(H-z_{n}\right)}{\beta}}\right]$

where the velocity parameter is defined by

$\beta \equiv 0.08552 \frac{g\left(\rho-\rho_{b}\right)}{\rho_{b}^{2 / 3} \mu}$

with units [length]/[time][mass $]^{2 / 3}$. 


\section{Mass Loss Rate}

The mass of a bubble exiting the surface depends upon its nucleation location. By the substitution of Eq. 11 into Eq. 8, the exiting bubble mass is

$$
m_{e}\left(z_{n}\right)=\left[m_{n}^{4 / 3}+\frac{2 \alpha\left(H-z_{n}\right)}{\beta}\right]^{3 / 4}
$$

Since the material properties are approximately constant over a bubbles lifespan, the nucleation rate in Eq. 1 can be approximated by $J\left(t-\tau_{b}\left(z_{n}\right)\right) \approx J(t)$. The validity of this assumption can be tested by comparison of the bubble lifespan with the characteristic times of pyrolysis, mass loss, and volatile amount evolution. Substituting Eq. 13 into Eq. 1 gives the final form of the bubbling loss model

$$
\frac{d m}{d t}=-J A_{s} H m_{e}
$$

where the average mass of the exiting bubbles are defined by

$$
\bar{m}_{e} \equiv \frac{2 \beta}{7 \alpha H}\left[\left(m_{n}^{4 / 3}+\frac{2 \alpha H}{\beta}\right)^{7 / 4}-m_{n}^{7 / 3}\right]
$$

\section{RELATION OF MODELS TO MATERIAL PROPERTIES}

Equations 4 and 14 together are a complete model for pyrolysis and devolatilization in TGA. However, as was discussed previously, the parameters of Eq. 14 are dependent on the state of the material. The material state is partly accounted for by the MW of the polymer as computed by Eq. 5, but the relative amount of the volatile species is also necessary. If the mass stored in bubbles at any given time is small relative to the total mass, then the volume fraction of the volatile species can be computed by

$$
\phi_{1}=\frac{\bar{v}_{1}}{M_{1} A_{s} H}\left(m-m_{2}\right)
$$

The height of the sample can be computed as $H=m / \rho A_{s}$, where $\rho$ is the mixture density. As will be seen in the next section, the mixture density is dependent upon the volume fraction of volatiles so it is necessary to solve for $H$ using the mixture density model and Eq. 16.

\section{MATERIAL PROPERTY DATA}

In this paper, the material of interest is high-density polyethylene (HDPE). The parameters in the loss model are difficult to characterize for polymeric materials - even structurally simple thermoplastics such as HDPE. In this section, experimental data and property models for HDPE are reviewed.

\section{Scission Rate}

Thermal degradation is initiated by chain scission. Many researchers have attempted to estimate scission rate from TGA results, but this approach neglects the rate at which small molecules devolatilize. It is hoped that the loss model developed in this paper can be used to obtain more accurate estimates of the overall scission rate from TGA - that is, the loss model can be used in parameter calibration.

An alternative use of the loss model is prediction. To make predictions of thermal degradation, it is necessary to have an estimate of the scission rate as an input to the models presented above. Full ab initio calculations of large molecules in condensed phases are computationally infeasible. A reasonable alternative that is partially fundamental is reactive molecular dynamics (RMD). In RMD, the classical equations of motion are integrated to predict atomic trajectories, but unlike classical molecular dynamics a 
reactive forcefield is employed. The reactive forcefield can be generated by using switching functions to smoothly transition from bonded to non-bonded interactions between atoms.

HDPE was studied using RMD in Ref. [14]. In this paper, $n$-alkanes of varying length were simulated until a first scission was observed. For the largest molecule studied, 200 backbone carbons, the activation energy and pre-exponential were found to be $251 \mathrm{~kJ} / \mathrm{mol}$ and $10^{14.6} 1 / \mathrm{s}$.

\section{Densities}

High-density polyethylene is, by definition, any polyethylene with a solid density of at least $9.41 \mathrm{~g} / \mathrm{cm}^{3}$. The density of polyethylene melts is around $20 \%$ less than this value. Bird et al. [15] cite data that can be linearly fit by the correlation

$\rho_{2}(T)=\left(1.158+8.09 \times 10^{-4} \mathrm{~T}\right)^{-1} \mathrm{~g} / \mathrm{cm}^{\mathrm{a}},{ } 133^{\circ} \mathrm{C}<T<200^{\circ} \mathrm{C}$

The density of smaller $n$-alkanes depends strongly on both MW and temperature. For temperatures between $20-100{ }^{\circ} \mathrm{C}$ and carbon numbers between 3-46, von Meerwall et al. [16] found that literature data from a variety of sources could be fit by

$\rho_{1}(T, M)=1 / \rho(T, M \rightarrow \infty)+2 V_{\theta}(T) / M$

where $V_{e}$ is the molar volume of the end-chain group. The authors provide correlations for the two parameters in Eq. 18 for $n$-alkanes. Equation 18 can be used for computing the density of binary mixtures if $M$ is replaced by the volume average MW.

\section{Notional Volatiles}

The volatile pyrolysis products of HDPE are various alkanes and alkenes distributed over a range of carbon numbers. The products appear to be smaller than $n_{c}=30$ for temperatures around $400{ }^{\circ} \mathrm{C}$ - the critical carbon number varies significantly with temperature. Plots of the carbon number distribution at these temperatures reveal that the average carbon number is around 12 . Therefore, in the simulations of the next section, the carbon number of the notional volatile species will be taken to be one-third of the critical carbon number.

\section{Critical Carbon Number}

There is a much experimental data on the vaporization properties of $n$-alkanes and $n$-alkenes. In particular, there are a number of correlations that relate the boiling temperature of these molecules to their carbon numbers. Such correlations can be inverted to give an estimate of the critical carbon number as a function of temperature. Inverting the correlation suggested by Egloff et al. [17] yields

$n_{e}(T)=10^{\frac{T+416.31}{745.42}}-4.4$

where $T$ is in units kelvin. Within the range of carbon numbers 2 to 19 , the model agrees with experimental data to within less than one percent.

\section{Surface Tension}

The nucleation rate depends strongly on the interfacial tension between the polymer melt and the bubble, $\sigma$. It is assumed that $\sigma$ is approximately the surface tension of the polymer melt in air for which there is data in the literature. $\mathrm{Wu}$ [18] performed pendent drop experiments to measure the surface tension of HDPE at temperatures of $300-450 \mathrm{~K}$. It was found that the temperature dependence is linear with

$$
\sigma(T)=0.0524-\left(5.7 \times 10^{-5}\right) T
$$




\section{Partial Pressures of Volatiles in Polymers: Flory-Huggins Theory}

Both the nucleation and growth models require information about the partial pressure of the volatile species in the polymer matrix. It was previously assumed that this partial pressure can be determined from Henry's law. In this section, it is shown how Flory-Huggins theory [9] can provide an estimate of the Henry's law coefficient. The fundamental quantity of this theory is the interaction parameter, $\chi$, that measures the degree to which the volatile is compatible with the polymer. A large $\chi$ indicates poor compatibility, and negative values correspond to good compatibility. Henry's law, expressed in terms of the interaction parameter, is

$$
P_{1}=K_{\phi} \phi_{1}=P_{1}^{0} e^{1+\chi} \phi_{1}
$$

where $P_{1}^{0}$ is the vapor pressure of the pure volatile.

Values of $\chi$ for linear alkanes in HDPE are available in the literature. Patterson et al. [19] report a value of $0.18+/-0.05$ for $n$-decane in a linear polyethylene at temperatures between $418-463 \mathrm{~K}$ and for small values of volatile volume fraction. Similar work by Brockmeier et al. [20] indicates that the dependence of the interaction parameter on volatile volume fraction is negligible for volume fractions between 0.01 and 0.18 . Within these concentration limits, the value of $\chi$ was found to be $0.12+/-0.05$ at a temperature of $458 \mathrm{~K}$. Schreiber et al. [21] measured $\chi$ for various hydrocarbons in HDPE at $422 \mathrm{~K}$. These experiments included results for $n$-alkanes with carbon numbers of $n=8,9,10$, and 12. The dependence of the interaction parameter on chain length is approximately linear: $\chi=-0.02 n+0.52$. The values reported by Schreiber et al. are significantly higher than the values from the other two sources. This could be due to a temperature dependence or to different analysis procedures.

The vapor pressures of $n$-alkanes have been extensively studied [22-25]. Ruzicka and Majer [25] surveyed the literature data before 1993. The results of this survey are summarized in terms of the coefficients of the Cox equation

$\ln \frac{P_{1}^{0}}{P^{0}}=\left(1-\frac{T_{b}}{T}\right) \exp \left[A_{0}\left(n_{1}\right)+A_{1}\left(n_{1}\right) T+A_{2}\left(n_{1}\right) T^{2}\right]$

where $P^{0}$ is the standard pressure. Within the range of $n$-alkanes considered in this survey $(n=5-20)$, the following approximations for the coefficients of Eq. 22 seem reasonable

$$
\begin{aligned}
& T_{b} \approx 20.1 n+235 \\
& A_{0} \approx 0.0367 n+2.60 \\
& A_{1} \approx-2.04 \times 10^{-3} \\
& A_{2} \approx-\left(7.13 \times 10^{-8}\right) n+\left(2.60 \times 10^{-6}\right)
\end{aligned}
$$

where temperature is in units kelvin.

\section{Molar Volume of the Volatile Species}

It is also necessary to specify the molar volume in order to compute Eq. 9. The required molar volume is the space occupied by a mole of the volatile molecules in the condensed phase matrix. Molar volumes for various solvents are provided in Ref. [9] at $0 \mathrm{~K}$. For the $n$-alkanes, the results are nearly exactly linear following

$$
\bar{v}_{1} \approx 14.0 n_{1}+15.3
$$

\section{Diffusivities}

Equation 9 depends upon the diffusivity of the notional volatile within the polymer matrix. The theory of multi-component diffusion is complex and difficult to characterize empirically. 
Diffusion in liquid $n$-alkanes and in $n$-alkane/polyethylene mixtures has been studied experimentally and theoretically by von Meerwall et al. [16,26]. It was found that the self-diffusion of $n$-alkanes in PE melts can be modeled by

$D_{1}\left(T, M_{1}, M_{2}, \phi_{1}\right)=\frac{A}{M_{1}} \exp \left(-\frac{E_{m}}{R T}-\frac{1}{f}\right)\left\{1+\frac{M_{1}}{\gamma\left[1+15\left(M_{C} / M_{1}\right)^{2]}\right]}\right\}^{-1}$

where $f$ is the free-volume fraction, $r$ is the ratio of reptation and Rouse diffusion coefficient constants, and $M_{c}$ is the critical molecular weight for chain entanglements. Also, $E_{a}=0.81 \mathrm{kcal} / \mathrm{mol}$ and $A=$ $0.306 \mathrm{~cm}^{2} \cdot \mathrm{mol} / \mathrm{g} \cdot \mathrm{s}$ where these constants were found from NMR experiments on $n$-alkanes. For binary mixtures,

$f\left(T, M_{1}, M_{2}, \phi_{1}\right)=f_{s e}(T)+2 V_{e}(T) \rho\left(T, M_{V}\right) / M_{V}$

where $M_{v}$ is the volume average molecular weight and $\rho$ is the mixture density. The equations for these three parameters are given above. In addition, von Meerwall provides correlations for the coefficient $r$ and the fractional free-volume at infinite MW.

\section{Viscosity}

The bubble velocity depends on the viscosity of the melt. Polymers are generally non-Newtonian showing a varying viscosity at high shear rates [15]. At low bubble velocities, the stress tensor can be approximated by Newton's law of viscosity thus making it necessary to specify a Newtonian viscosity only. This viscosity is strongly dependent on the size distributions of the polymer chains. Berry and Fox [27] studied the viscosity of many polymer melts and found that

$\log \mu\left(M_{w}, T\right)= \begin{cases}\log \mu_{\theta}(T)+\log \left(M_{w} / M_{\theta}\right), & M_{w}<M_{\theta} \\ \log \mu_{\theta}(T)+3.4 \log \left(M_{W} / M_{\theta}\right), & M_{w}>M_{\theta}\end{cases}$

where $M_{w}$ is the weight average molecular weight of the melt and $\mu_{e}$ and $M_{e}$ are the entanglement viscosity and molecular weight.

\section{Nucleation Rate and Bubble Size}

Predictions of the nucleation rate and nucleation bubble size are difficult. In order to provide estimates of these quantities as inputs for a TGA simulation, a simple experiment was performed. In this experiment, a small sample of HDPE pellets was pyrolyzed in a test tube. The sample temperature was maintained between $390-420^{\circ} \mathrm{C}$. Pictures were taken to obtain information about bubble size. Figure 2 shows the sample before it was melted and then at three times after it melted and began to pyrolyze. At time $t_{0}$, the melted portions are clear, but there are still significant regions of solid phase HDPE. It was observed that there were relatively large bubbles formed during this time period possibly due to gas accumulating under un-melted material. The next snapshot was taken two minutes later. At this point the noticeable features are (1) many more bubbles, (2) increased bubble velocity, (3) a change yellowish tint to the melt, and (4) the formation of a bubble 'fizz' layer at the surface of the melt. The change in color is thought to be due to a significant change in average MW of the material. This hypothesis was somewhat confirmed by examining the re-solidified material which was waxy and opaque. The final snapshot was taken four minutes after $t_{0}$ and seems to be qualitatively similar to the previous picture. In fact the only noticeable differences are a decreased sample height and a further darkening of color. Most nucleation appears to be heterogeneous and at the bottom of the test tube. The vast majority of bubbles seemed to form on the bottom of the test tube. 


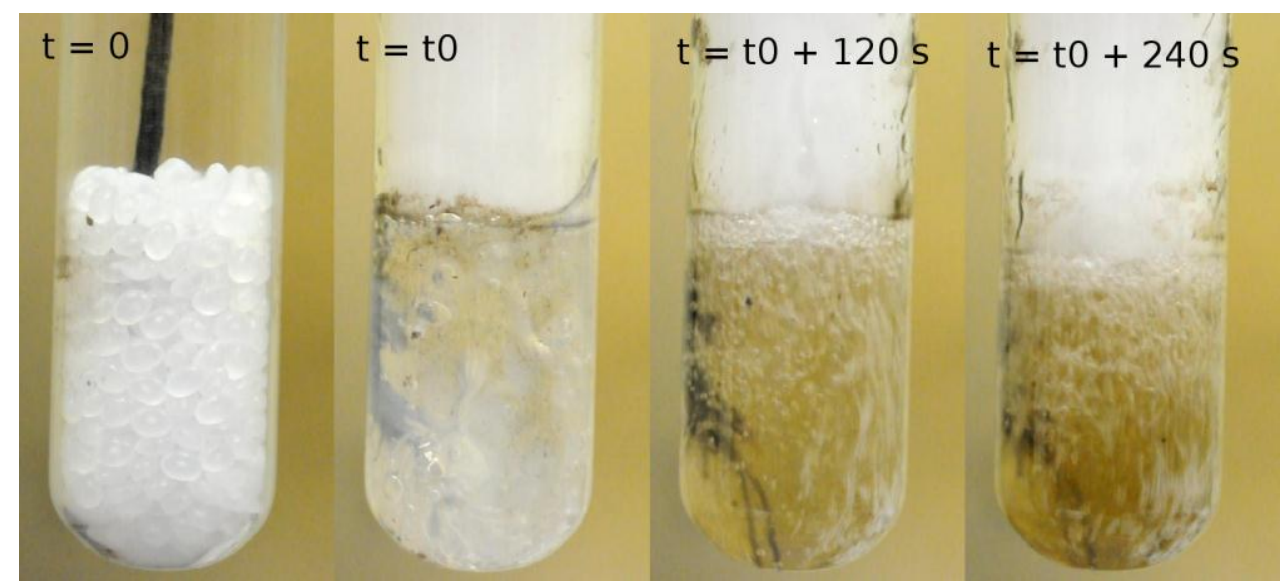

Fig. 2. Pyrolysis of HDPE at $T \approx 400^{\circ} \mathrm{C}$.

The third picture in Fig. 2 was digitized and many bubble diameters were measured. The bubble size distribution is plotted in Fig. 3. The average bubble diameter was approximately $0.6 \mathrm{~mm}$-a value which conforms to other observations (see, e.g. Ref. [1]). Unfortunately, the camera used in these experiments was not fast enough to accurately measure bubble velocities.

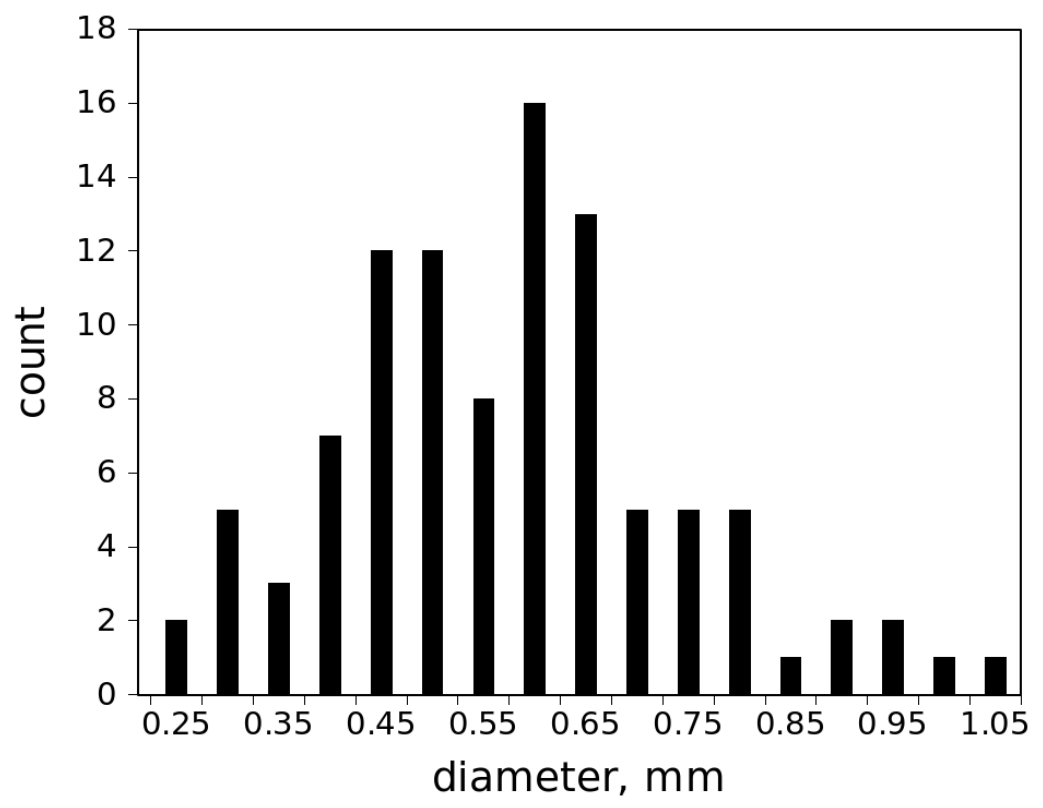

Fig. 3. Bubble size distribution in pyrolyzing HDPE at $400{ }^{\circ} \mathrm{C}$.

\section{RESULTS AND DISCUSSION}

Simulations were performed by integrating Eq. 14 using material properties based upon the data of the previous section. The input parameter values used are listed in Tables 1-3. Scenario parameters (Table 1) were chosen to conform to typical isothermal TGA operating conditions. Table 2 contains a list of all of the material properties that were modeled as having only temperature dependence and were therefore constant throughout the simulation. Table 3 lists minimum, mean, and maximum values for the material properties that varied dynamically due to their dependence on the melt composition. 
Table 1. List of scenario parameters.

\begin{tabular}{|c|l|}
\hline$T$ & $410^{\circ} \mathrm{C}$ \\
\hline$m_{i}$ & $10 \mathrm{mg}$ \\
\hline$M_{2, i}$ & $13.9 \mathrm{~kg} / \mathrm{mol}$ \\
\hline$A_{s}$ & $8 \mathrm{~mm}^{2}$ \\
\hline
\end{tabular}

Table 2. List of constant (i.e. independent of composition) material properties.

\begin{tabular}{|c|l|}
\hline$k_{s}$ & $9.64 \mathrm{e}-6 \mathrm{~s}^{-1}$ \\
\hline$n_{c}$ & 25.5 \\
\hline$n_{1}$ & 8.48 \\
\hline$P_{1}{ }^{0}$ & $11.2 \mathrm{kPa}$ \\
\hline $\bar{v}_{1}$ & $134.1 \mathrm{~cm}^{3} / \mathrm{mol}$ \\
\hline$\chi$ & 0.35 \\
\hline$\sigma$ & $13.5 \mathrm{mN} / \mathrm{m}$ \\
\hline$M_{1}$ & $0.121 \mathrm{~kg} / \mathrm{mol}$ \\
\hline$r_{n}$ & $0.5 \mathrm{~mm}$ \\
\hline
\end{tabular}

Table 3. List of composition dependent material properties.

\begin{tabular}{|c|l|l|l|}
\hline & \multicolumn{1}{|c|}{ Minimum } & \multicolumn{1}{c|}{ Mean } & \multicolumn{1}{c|}{ Maximum } \\
\hline$\mu, \mathrm{kg} / \mathrm{m}-\mathrm{s}$ & 0.0002 & 2.2185 & 24.3938 \\
\hline$D_{1}, \mathrm{~cm}^{2} / \mathrm{s}$ & $1.08 \mathrm{e}-4$ & $2.09 \times 10^{-4}$ & $2.58 \times 10^{-4}$ \\
\hline$J, 1 / \mathrm{cm}^{3} \cdot \mathrm{s}$ & 0 & 34.2 & 52.6 \\
\hline$\alpha, \mathrm{kg}^{2 / 3} / \mathrm{s}$ & 0 & $2.11 \times 10^{-5}$ & $4.13 \times 10^{-5}$ \\
\hline$\beta, \mathrm{m} / \mathrm{kg}^{2 / 3} \cdot \mathrm{s}$ & 0 & $0.8721 \times 10^{6}$ & $1.68 \times 10^{6}$ \\
\hline
\end{tabular}

The results of these simulations are plotted in Figs. 4 and 5. Experimental data from Conesa et al. [28] was used for comparison. Figure 4 shows normalized masses as a function of time. The volatile mass is represented by the dashed line, the polymer mass is represented by the dotted line, the total mass (sum of volatile and polymer) is represented by the solid line, and the mass measurements from a TGA experiment are represented by the circles. The bubbling loss model introduces a lag into the mass loss rate. For the system studied, the inclusion of this lag seems to improve the prediction up until around $150 \mathrm{~min}$. At this point, the bubble loss model under predicts the mass loss rate indicating that the diffusion of volatiles to the surface is becoming significant. It is observed that the point of divergence between the experiment and the model corresponds to the point at which the sample becomes mostly composed of volatiles. Thus, at large times, the bubble loss model needs to be supplemented by a surface evaporation loss model.

Figure 5 shows normalized lengths as a function of time. The sample initially swells as the polymer is converted to a lower MW liquid. This is due to the increasing free-volume associated with a higher number of chain end groups (see Eq. 18). Bubble nucleation does not begin until approximately 30 min into the simulation. It is observed that the bubbles do not grow significantly as they migrate to the surface. At around $210 \mathrm{~min}$ the sample becomes thinner than a single bubble diameter, and the model is obviously no longer valid.

In Fig. 6 are three TGA traces from Ref. [28] along with predictions based upon the bubbling loss model. At temperatures of $400{ }^{\circ} \mathrm{C}$ and $410{ }^{\circ} \mathrm{C}$, the model predicts the experimental data fairly well. The model begins to under predict the mass loss rate at $410{ }^{\circ} \mathrm{C}$ for high conversions $\left(m^{*}<0.4\right)$. This trend becomes especially noticeable for the simulation at $420^{\circ} \mathrm{C}$. This divergence between model and experiment is most likely due to changes in the material properties due to temperature that were not properly accounted for. Another possible explanation for the insufficiency of the model at higher temperatures is an increase in volatile diffusion directly to the sample surface. 


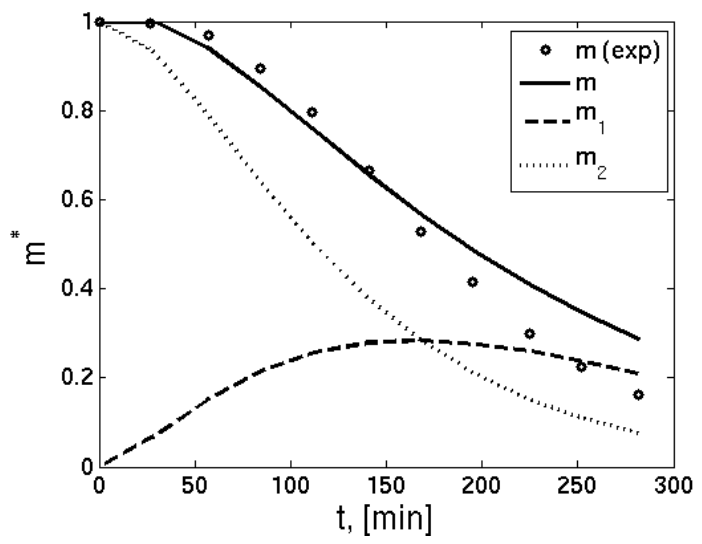

Fig. 4. TGA simulation of HDPE and experimental data at $T=410^{\circ} \mathrm{C}$.

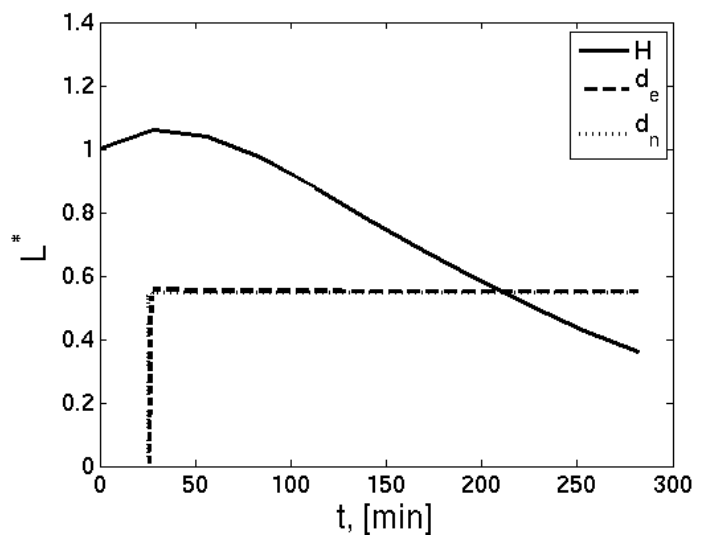

Fig. 5. Sample height and bubble diameters in TGA simulation of HDPE at $T=410^{\circ} \mathrm{C}$.

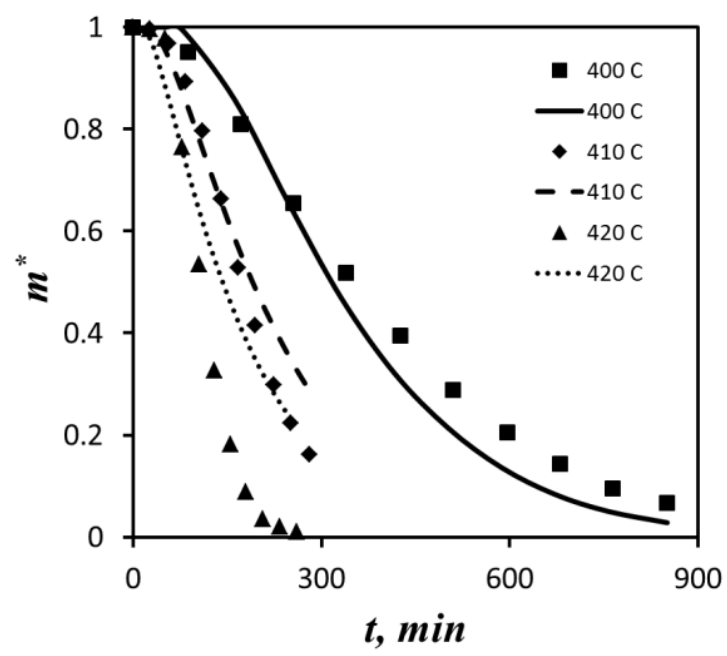

Fig. 6. TGA simulation of HDPE at several temperatures (markers represent experimental data).

\section{CONCLUSIONS}

A simple model of TGA has been developed-Objective (1). This includes pyrolysis, modeled as random scission, and devolatilization, modeled as bubble nucleation, growth, and migration across the sample surface. The bubble loss mechanism seems promising as a model for predicting TGA data for linear 
thermoplastics, but the model has a limited range of validity and needs to be supplemented by a diffusion and surface vaporization loss mechanism.

Making predictions based on this model required a brief literature review of HDPE material propertiesObjective (2). There is a great deal of uncertainty in the material property estimates, and it would be helpful to perform a sensitivity analysis to find which properties need to be more accurately modeled.

Finally, the model was compared to experimental TGA data for HDPE-Objective (3). It seems that the model can predict TGA traces fairly well at low temperatures. In order to extend the applicability of the model to higher temperatures, it will be necessary to improve the material property models.

\section{ACKNOWLEDGEMENTS}

The authors would like to thank Reed Anzalone for help with the pyrolysis experiments. This material is based upon work supported by the Department of Energy (National Nuclear Security Administration) under Award Number (DE-FC52-08NA28615).

\section{REFERENCES}

[1] Wichman, I.S., (1986) A Model Describing the Steady-State Gasification of Bubble-Forming Thermoplastics in Response to an Incident Heat Flux, Combustion and Flame 63: 217-229, http://dx.doi.org/10.1016/0010-2180(86)90122-7

[2] Faravelli, T., Bozzano, G., Scassa, C., Perego, M., Fabini, S., Ranzi, E., and Dente, M., (1999) Gas Product Distribution from Polyethylene Pyrolysis, Journal of Analytical and Applied Pyrolysis 52: 87-103, http://dx.doi.org/10.1016/S0165-2370(99)00032-7

[3] Butler, K.M., "A Numerical Model for Combustion of Bubbling Thermoplastic Materials in Microgravity," National Institute of Standards and Technology Report NISTIR 6894, Gaithersburg, MD, 2002.

[4] Conley, R.T. (ed.), Thermal Stability of Polymers, Vol. 1, Marcel Dekker, Inc., New York, 1970.

[5] Grassie, N. and Scott, G., Polymer Degradation and Stabilisation, Cambridge University Press, New York, 1985.

[6] Jellinek, H.H.G. (ed.), Aspects of Degradation and Stabilization of Polymers, Elsevier Scientific Publishing Company, New York, 1978.

[7] Bruns, M.C., Koo, J.H., and Ezekoye, O.A., (2009) Population-based Models of Thermoplastic Degradation: Using Optimization to Determine Model Parameters, Polymer Degradation and Stability 94: 1013-1022, http://dx.doi.org/10.1016/j.polymdegradstab.2009.02.007

[8] Blander, M. and Katz, J.L., (1975) Bubble Nucleation in Liquids, AIChE Journal 21: 833-848, 1975, http://dx.doi.org/10.1002/aic.690210502

[9] Albalak, R.J. (ed.), Polymer Devolatilization, Marcel Dekker, Inc., New York, 1996.

[10] Kwak, H.Y. and Kim, Y.W., (1998) Homogeneous Nucleation and Macroscopic Growth of Gas Bubble in Organic Solutions, International Journal of Heat and Mass Transfer 41: 757-767, http://dx.doi.org/10.1016/S0017-9310(97)00182-8

[11] Yarin, A.L., Lastochkin, D., Talmon, Y., and Tadmor, Z., (1999) Bubble Nucleation During Devolatilization of Polymer Melts, AIChE Journal 45: 2590-2605, http://dx.doi.org/10.1002/aic.690451216

[12] Kim, K.Y., Kang, S.L., and Kwak, H.Y., (2004) Bubble Nucleation and Growth in Polymer Solutions, Polymer Engineering and Science 44: 1890-1899, http://dx.doi.org/10.1002/pen.20191.

[13] Epstein, P.S. and Plesset, M.S., (1950) On the Stability of Gas Bubbles in Liquid-Gas Solutions, Journal of Chemical Physics 18: 1505, http://dx.doi.org/10.1063/1.1747520 
[14] Smith, K.D., Bruns, M.C., Stoliarov, S.I., Nyden, M.R., Ezekoye, O.A., and Westmoreland, P.R., (2011, in review) A New Reactive Molecular Dynamics Method, Demonstrated by Predicting Kinetics of Backbone Scission in Polyethylene, Polymer.

[15] Bird, R.B., Armstrong, R.C., and Hassager, O., Dynamics of Polymeric Liquids, Volume 1 Fluid Mechanics, John Wiley and Sons, New York, 1987.

[16] von Meerwall, E., Beckman, S., Jang, J., and Mattice, W.L., (1998) Diffusion of $n$-alkanes: Freevolume and Density Effects, Journal of Chemical Physics 108: 4299-4304, http://dx.doi.org/10.1063/1.475829

[17] Egloff, G., Sherman, J., and Dull, R.B., (1940) Boiling Point Relationships Among Aliphatic Hydrocarbons, The Journal of Physical Chemistry 44: 730-745, http://dx.doi.org/10.1021/j150402a006

[18] Wu, S., (1969) Surface and Interfacial Tensions of Polymer Melts: I. Polyethylene, Polyisobutylene, and Polyvinyl Acetate, Journal of Colloid and Interface Science 31: 153-161, http://dx.doi.org/10.1016/0021-9797(69)90321-X

[19] Patterson, D., Tewari, Y.B., Schreiber, H.P., and Guillet, J.E., (1971) Application of Gas-Liquid Chromatography to the Thermodynamics of Polymer Solutions, Macromolecules 4: 356-359, http://dx.doi.org/10.1021/ma60021a023

[20] Brockmeier, N.F., McCoy, R.W., and Meyer, J.A., (1972) Gas Chromatographic Determination of Thermodynamic Properties of Polymer Solutions, Macromolecules 5: 130-132, http://dx.doi.org/10.1021/ma60026a006

[21] Schreiber, H.P., Tewari, Y.B., and Patterson, D., (1973) Thermodynamic Interactions in Polymer Systems by Gas-Liquid Chromatography. III. Polyethyelene-hydrocarbons, Journal of Polymer Science: Polymer Physics Edition 11: 15-24.

[22] Chickos, J.S., (2004) Vapor Pressures and Vaporization Enthalpies of the $n$-alkanes from $C_{21}-C_{30}$ at $T=298.15 \mathrm{~K}$ by Correlation Gas Chromatography, Journal of Chemical \& Engineering Data 49: 77-85.

[23] Chirico, R.D., Nguyen, A., V.S., Steele, and Strube, M.M., (1989) Vapor Pressure of $n$-alkanes Revisited. New High-precision Vapor Pressure Data on $n$-decane, $n$-eicosane, and $n$-octacosane, Journal of Chemical \& Engineering Data 34: 149-156, http://dx.doi.org/10.1021/je00056a00

[24] Gallant, R.W. and Yaws, C.L., Physical Properties of Hydrocarbons, Gulf Publishing Company, Houston, 1992.

[25] Ruzicka, K. and Majer, V., (1994) Simultaneous Treatment of Vapor Pressures and Related Thermal Data Between the Triple and Normal Boiling Temperatures for $n$-alkanes $C_{5}-C_{20}$, Journal of Physical and Chemical Reference Data 23: 1-39, http://dx.doi.org/10.1063/1.555942

[26] von Meerwall, E., Feick, E.J., Ozisik, R., and Mattice, W.L., (1999) Diffusion in Binary Liquid nalkane and Alkane-polyethylene Blends, Journal of Chemical Physics 111: 750-757, http://dx.doi.org/10.1063/1.479354

[27] Berry, G.C. and Fox, T.G., (1968) The Viscosity of Polymers and their Concentrated Solutions, Advances in Polymer Science 5: 261-357.

[28] Conesa, J.A., Marcilla, A., Font, R., and Caballero, J.A., (1996) Thermogravimetric Studies on the Thermal Decomposition of Polyethylene, Journal of Analytical and Applied Pyrolysis 36: 1-15, http://dx.doi.org/10.1016/0165-2370(95)00917-5 